\title{
An analysis of the beam interaction characteristics of selected lasers with an alpha-alumina bioceramic
}

\author{
J. Lawrence
}

Manufacturing Engineering Division, School of Mechanical \& Production Engineering, Nanyang Technological University (NTU), Nanyang Avenue, Singapore 639798.

\section{Correspondence}

Dr. Jonathan Lawrence

Manufacturing Engineering Division,

School of Mechanical \& Production Engineering,

Nanyang Technological University (NTU),

Nanyang Avenue,

Singapore 639798.

Tel : (+65) 67905542

Fax : (+65) 67911859

e-mail : mjlawrence@ntu.edu.sg 


\begin{abstract}
Certain differences between the interaction characteristics of a $\mathrm{CO}_{2}$ laser, a Nd:YAG laser and a high power diode laser (HPDL) with an alpha-alumina bioceramic have been investigated. For each laser the fluence threshold values at which significant material removal occurs were found graphically. Through the implementation of a derivative of Beer-Lambert's law, the laser beam absorption lengths were calculated along with the thermal loading values. An examination of the laser induced meltpool propagation in the alpha-alumina revealed good agreement with the Stefan solution to the heat diffusion equation for the lasers. Absorptivity measurements revealed that there was no correlation between the actual absorptivity of the alpha-alumina and the absorption length for each of the three lasers on account of the absorptivity measurements being similar for each laser. However, differences in the depth of melting experienced by the alpha-alumina meant that it is reasonable to assume that absorption length is the principal influence on the melt depth.
\end{abstract}

Keywords: Laser; Interaction characteristics; alpha-Alumina; Bioceramic 


\section{Introduction}

On account of its great strength and high hardness, alumina, particularly alpha-alumina, is finding increasing usage in modern surgery for a variety of purposes including: hip ball \& cup replacements; knee joint implants and bone screws. Likewise in dentistry, alpha-alumina is used to fabricate dental implants as well as crowns and brackets [1]. Alpha-alumina is a bioceramic of an inert nature, that is it does not naturally form a bonding to bone. Nevertheless, its excellent in vivo corrosion and wear resistance afford it high usage. In contrast, application of bioactive ceramics and glasses which are capable of sustaining, and perhaps promoting, tissue bonding remain limited on account of their tendency to fracture under load-bearing conditions $[2,3]$. Clearly it would be highly beneficial if some degree of bioactivity could be induced into alpha-alumina since it is eminently the implant material of choice. To this end certain surface treatments (roughening) have been tried but have proved to be less effective than was hoped for [4]. It is conceivable, however, that laser treatment could provide an effective alternative. Indeed, work by Lawrence and Li has shown that laser surface treatment of ceramic [5], metallic [6,7] and polymeric [8] materials can improve their wettability characteristics towards biological liquids such as blood and blood plasma. By using a $\mathrm{CO}_{2}$, a Nd:YAG and a HPDL this paper aims to investigate the supposition that the absorption characteristics (in terms of laser wavelength variations) of the alpha-alumina may play a significant role in influencing the depth to which laser-induced microstructural changes occur.

Fundamental differences in the general laser interaction characteristics experienced by materials when interacting with different lasers is an aspect of great significance, since the end results of many laser processing procedures are governed by this parameter. Although only few in number, previous studies into the effects of the laser wavelength variations of materials processing lasers such as $\mathrm{CO}_{2}$, Nd:YAG and excimer lasers, and more recently the high power diode laser (HPDL), have revealed distinct differences in performance. In an early study Dausinger [9] compared $\mathrm{CO}, \mathrm{CO}_{2}$ and $\mathrm{Nd}: \mathrm{YAG}$ lasers for a number of materials processing applications, noting differences resulting from wavelength variations. From work using $\mathrm{CO}_{2}$ and excimer lasers to examine the effect of laser wavelength on the ablation of soda-lime-silica glass, Shuttleworth [10] observed distinct differences in the performance of the lasers in terms of process effectiveness. Similarly, Dyer et al [11] found that laser wavelength was a major influence in determining the likelihood of successful deposition whilst investigating alternative routes to the laser ablation-deposition (LAD) of $\mathrm{Al}_{2} \mathrm{O}_{3}$ and Ti:saphire using $\mathrm{CO}_{2}$ and excimer radiation. A study conducted by Chen et al [12] using Nd:YAG and excimer 
lasers found that the quality of laser drilled holes was influenced crucially by laser wavelength. Both Bradley et al [13] and Lawrence and $\mathrm{Li}[14,15]$ have remarked on the generation of wavelength dependant microstructural characteristics on $\mathrm{Al}_{2} \mathrm{O}_{3}$-based refractories and the ordinary Portland cement (OPC) surface on concrete respectively when using $\mathrm{CO}_{2}$ and HPDLs.

\section{Experimental procedures}

This work was conducted using a $\mathrm{CO}_{2}$ laser (Rofin-Sinar, DC 015), a Nd:YAG laser (Lumonix, JK704) and a GaAlAs HPDL (Rofin-Sinar, DL 015). The $1.5 \mathrm{~kW} \mathrm{CO}_{2}$ laser beam was focused onto the surface of the alpha-alumina samples through a $200 \mathrm{~mm}$ focal length $\mathrm{KC} 1$ lens to give a stable diverging beam. For the $400 \mathrm{~W} \mathrm{Nd:YAG} \mathrm{laser,} \mathrm{the} \mathrm{beam} \mathrm{was} \mathrm{delivered} \mathrm{to} \mathrm{the} \mathrm{surface} \mathrm{of} \mathrm{the} \mathrm{alpha-}$ alumina samples by means of a $3 \mathrm{~m}$ long, $1 \mathrm{~mm}$ core diameter optical fibre. The beam, which was of a Gaussian profile, was then focused on to the surface of the samples by means of a $120 \mathrm{~mm}$ focal length focusing lens assembly. The $1.5 \mathrm{~kW}$ HPDL beam was delivered to the surface of the alphaalumina samples by means of a $5 \mathrm{~m}$ long, $1.5 \mathrm{~mm}$ core diameter optical fibre, the end of which was connected to a focusing lens assembly with a focal length of $42 \mathrm{~mm}$. In all three instances the laser optics were protected by means of a coaxially blown Ar shield gas jet a rate of $51 / \mathrm{min}$.

The alpha-alumina is studied in this work was the type typically used for hip ball \& cup replacements. For the purpose of experimental convenience the alpha-alumina was fabricated into blocks $\left(25 \times 25 \times 5 \mathrm{~mm}^{3}\right)$ prior to laser treatment. The alpha-alumina used was $99.4 \%$ pure $\mathrm{Al}_{2} \mathrm{O}_{3}$ with impurities of $\mathrm{CaO}, \mathrm{Fe}_{2} \mathrm{O}_{3}, \mathrm{~K}_{2} \mathrm{O}, \mathrm{MgO}, \mathrm{Na}_{2} \mathrm{O}, \mathrm{SiO}_{2}$ and $\mathrm{TiO}_{2}$ constituting the remaining $0.6 \%$.

Two sets of laser processing experiments were conducted. The first set of experiments were carried out in order to determine the minimum threshold fluence, the absorption length and to examine the thermal loading characteristics of the alpha-alumina. Here it was necessary that the fluences of all three lasers were set such that no melting occurred. The absence of melting and vitrification was verified by subjecting the irradiated areas to an X-ray diffraction (XRD) analysis to ensure the alphaalumina retained its crystallinity. So as to examine the meltpool generation characteristics of the alpha-alumina, a separate set of experiments were conducted in which the parameters of the lasers were set such that melting of the material was effected. The development of the accompanying meltpools were observed with a high speed motion analyser (Kodak, SR-Ultra-c) recording at a rate of 5000 frames per second (fps). 


\section{Minimum threshold fluence, beam absorption length and thermal loading}

The depth of material removed per pulse or shot, $h$, for many materials has been frequently described by a logarithmic law which follows, heuristically, from Beer-Lambert's law [16]

$$
h=\frac{1}{\alpha} \ln \left(\frac{F}{F_{t}}\right)
$$

where $\alpha$ is the optical absorption coefficient, $F$ is the laser fluence and $F_{t}$ is the threshold value of the fluence at which significant material removal occurs. It should be noted that (1) does not take into account any absorption of incident radiation by the plume of removed material and probable changes in the absorption level of the material as removal progresses. Nonetheless, as the work of Andrew et al [17], Schmidt et al [18] and Lawrence et al [19-21] demonstrated, this simple form of the expression is quite satisfactory as a first approximation.

The relationship between $h$ and $F$ for the $\mathrm{CO}_{2}$ laser, the Nd:YAG laser and the HPDL incident with the alpha-alumina are given in Fig. 1, Fig. 2 and Fig. 3 respectively. As one can see from these Figs., $h$ for the alpha-alumina irradiated with all three lasers was seen to display a logarithmic dependence on $F$. Furthermore, by using an optical profiling system (ProScan, Nanofinder) to determine the values of $h$ for the various values of $F$, the minimum required threshold fluence for discernible material removal, $F_{t}$, for each laser was determined from Figs. 1 - 3. These values for $F_{t}$ are given in Table 1. This approach is widely recognised as being sound for the examination of pulsed lasers (excimer) [16]. However, the technique has also been used to good effect to investigate continuous wave $(\mathrm{CW})$ lasers [18-21]. What is more, the distinct linearity of the data points for the $\mathrm{CO}_{2}$ and HPDLs in Fig. 1 and Fig. 3 further validates the use of this approach for the examination of CW lasers. It is important to note that this would assume a minimum conduction loss. However, the alphaalumina has a relatively low thermal conductivity $(18 \mathrm{~W} / \mathrm{mK})$, thus it is reasonable to assume that the laser power densities used were high enough and that the shots were short enough to minimise the thermal conduction loss.

Now, the term $1 / \alpha$ is the absorption length, so by rearranging (1) thus:

$$
\frac{1}{\alpha}=\frac{h}{\ln \left(\frac{F}{F_{t}}\right)}
$$


then it is possible to determine the length to which the beams of all three lasers are absorbed by the alpha-alumina. By introducing $h$ and the corresponding value of $F$ for each data point into (2) it was possible to calculate the average absorption length for each laser under the actual experimental conditions. These values calculated for $1 / \alpha$ are given in Table 1 . From a comparison of the absorption length values with the wavelengths at which each of the three lasers emit $\left(10.6 \mu \mathrm{m}\right.$ for the $\mathrm{CO}_{2}$ laser, $1.06 \mu \mathrm{m}$ for the Nd:YAG laser and $940 \mathrm{~nm}$ for the HPDL), one can see that the absorption length appears to be proportional to the laser wavelength. It is interesting at this point to consider the actual absorptivity value of the surface of the alpha-alumina. Absorptivity measurements made at room temperature using a comparative technique detailed elsewhere $[21,22]$ are given in Table 1 . As these figures are of a similar order, it is appears that there is no correlation between the actual absorptivity of the alpha-alumina and the absorption length for each of the lasers. Although at this point it is not possible to determine which one plays the primary role.

In studies conducted by Dyer et al [23, 24], the surface energy density or thermal loading, $\gamma$, of a material has been found to increases in an almost linear manner until the minimum threshold fluence, $F_{t}$, is attained. Beyond this point the thermal loading becomes almost constant due to the rapid ablation of products which carry away excess energy and consequently limit the maximum temperature rise. The value of $\gamma$ necessary to effect removal is given by [23]

$$
\gamma=F_{t} \alpha
$$

From a rearrangement of (2) it is possible to calculate the value of $\alpha$ for each laser (see Table 1). So, by introducing the values of $F_{t}$ for each of the lasers deduced earlier from Fig. 1 along with the values of $\alpha$ for each laser into (3), then $\gamma$ for the three lasers can be calculated (see Table 1). The relatively high values of $\gamma$ calculated for the lasers can be attributed to the fact that the alpha-alumina has, under certain laser operating parameters, a propensity for melting (and subsequent vitrification on resolidification) at the wavelengths that these lasers emit at [22].

\section{Meltpool generation characteristics and depth of melting}

From a consideration of the Stefan problem it can be seen that the propagation speed of a melt/solid interface, $v_{m / s}$, should be a constant and can be defined by 


$$
v_{m / s}=\frac{I_{a}}{\rho \Delta H}
$$

where $I_{a}$ is the absorbed laser intensity, $\rho$ is the density of the material and $\Delta H$ is the latent heat of melting for the material. It can also be deduced from (4) that the melt/solid interface propagation speed, $v_{m / s}$, is related linearly to the irradiance of the interaction zone (laser beam spot size). Indeed, Figs. 4 - 6 reveal that in this work there is good agreement with this observation. It is evident from these Figs. that the melt/solid interface propagation speeds for the lasers increase as irradiance increases. Furthermore, from Figs. 4 - 6 the meltpool propagation speeds for the lasers can be deduced (see Table 1). Indeed, if one considers Fig. 4 then it can be deduced that the meltpool propagation speeds for the $\mathrm{CO}_{2}$ laser are $0.73 \% \mathrm{~m} / \mathrm{s}$ at $2.2 \mathrm{~kW} / \mathrm{cm}^{2}$ irradiance and $0.87 \% \mathrm{~m} / \mathrm{s}$ at 2.8 $\mathrm{kW} / \mathrm{cm}^{2}$ irradiance. Hence an increase in irradiance of $26 \%$ yielded an increase of $19 \%$ in the meltpool propagation speed. Similar results were obtained for the Nd:YAG and HPDLs (see Table 1), where an increase in Nd:YAG laser irradiance of $56 \%$ brought about an increase of $53 \%$ in the meltpool propagation speed (see Fig. 5), and an increase in HPDL irradiance of $28 \%$ brought about an increase of $20 \%$ in the meltpool propagation speed (see Fig. 6). It is worth remarking that the Stefan problem assumes the reflectivity of the meltpool to be $100 \%$, implying that the laser radiation is not absorbed over the melt area; since assuming otherwise would prohibit a simple solution of (4). All the same, these findings show that the meltpool propagation correlates well with the Stefan solution to the heat diffusion equation.

These distinct differences observed in the general interaction characteristics (the minimum threshold fluence, beam absorption length and the thermal loading), along with the meltpool generation characteristics have significant practical implications. This is because in any surface treatment of the alpha-alumina, the depth to which the material melts and subsequently partially vitrifies is a crucial factor in determining the subsequent in vivo performance of the material. Therefore, experiments were conducted to investigate the effects of the general interaction characteristics and the meltpool generation characteristics of each laser on the melt depth. Here the incident beams of the lasers were traversed across the surface of the alpha-alumina samples, thus generating a single melt track. In all instances the power density was fixed at $450 \mathrm{~W} / \mathrm{cm}^{2}$ whilst the traverse speed was set at 1200 $\mathrm{mm} / \mathrm{min}$. As one can see clearly from Table 1, the maximum depth of melting was achieved when employing the $\mathrm{CO}_{2}$ laser, whilst the melt depths obtained when employing the $\mathrm{Nd}: \mathrm{YAG}$ and the HPDLs were over $50 \%$ less. It is interesting to note that the melt depths for the Nd:YAG and the 
HPDLs were very similar in value. This marked difference in melt depth between the $\mathrm{CO}_{2}$ laser and the other two laser is perhaps surprising when one considers the thermal diffusion length, $z_{t}$, which is given by

$$
z_{t}=2 \sqrt{a t}
$$

where, $a$, is the thermal diffusivity of the material and, $t$, is the beam material interaction time. Clearly, according to (5), the thermal diffusion length, and hence melting, is a function of the material. Therefore some other factor must come into play in order to cause the differences in the melt depth experienced by the alpha-alumina when exposed to radiation of the three lasers. The two most prominent factors that may influence the melt depth differences are arguably the absorption length, $1 / \alpha$, and the actual absorptivity of the alpha-alumina. Absorptivity measurements made at room temperature revealed that the alpha-alumina absorbed around $70 \%$ of $\mathrm{CO}_{2}$ laser radiation and around $65 \%$ and $61 \%$ of the Nd:YAG and HPDL radiation respectively. Since these figures are similar, it is perhaps reasonable to assume that the absorption length is the primary influence on the melt depth.

\section{Conclusions}

By making use of a derivative of Beer-Lambert's law, the laser beam absorption lengths of $\mathrm{CO}_{2}$ laser, Nd:YAG laser and HPDL radiation for an alpha-alumina were determined. The absorption lengths, $1 / \alpha$, were calculated as being $303 \pm 23 \mu \mathrm{m}$ for the $\mathrm{CO}_{2}$ laser, $106 \pm 7 \mu \mathrm{m}$ for the $\mathrm{Nd}: \mathrm{YAG}$ laser and $88 \pm 4 \mu \mathrm{m}$ for the HPDL. The fluence threshold values at which significant material removal occurs, $F_{t}$, for the $\mathrm{CO}_{2}$ laser, Nd:YAG laser and HPDL were deduced as being $12.36 \mathrm{~J} / \mathrm{cm}^{2}, 73.89 \mathrm{~J} / \mathrm{cm}^{2}$ and $83.43 \mathrm{~J} / \mathrm{cm}^{2}$ respectively. The thermal loading, $\gamma$, value for the $\mathrm{CO}_{2}$ laser, the Nd:YAG laser and the HPDL were determined as $397.92 \mathrm{~J} / \mathrm{cm}^{3}, 6.65 \mathrm{~kJ} / \mathrm{cm}^{3}$ and $9.19 \mathrm{~kJ} / \mathrm{cm}^{3}$ respectively. It is maintained that the relatively high values of $\gamma$ observed are due to the tendency of the alpha-alumina for melting (and subsequent vitrification on resolidification) at the wavelengths at which the $\mathrm{CO}_{2}, \mathrm{Nd}: \mathrm{YAG}$ and HPDLs emit. An analysis of the laser induced meltpool propagation in the alpha-alumina revealed good agreement with the Stefan solution to the heat diffusion equation for the $\mathrm{CO}_{2}, \mathrm{Nd}$ :YAG and HPDLs. Absorptivity measurements revealed that there was no correlation between the actual absorptivity of the alpha-alumina and the absorption length for each of the three lasers on account of the absorptivity measurements being similar for each laser. However, differences in the depth of 
melting experienced by the alpha-alumina meant that it is reasonable to assume that absorption length is the principal influence on the melt depth.

\section{References}

1. Li J, Hastings GW. "Oxide bioceramics: Inert ceramic materials in medicine and dentistry" in Black J and Hastings G W (Eds.) Handbook of Biomaterial Properties (London: Chapman \& Hall) 1998, pp. 340-53

2. Santos JD, Morrey S, Hastings GW, Monteiro FJ. "The production and characterisation of a hydroxyapatite ceramic material" in Bonfield W, Hastings G W and Tanner K E (Eds.) Bioceramics (London: Butterworth-Heinemann) 1991, pp. 71-8

3. Planell JA, Vallet-Regi M, Fernandez E, Rodriguez LM. "Fracture toughness evaluation of sintered hydroxyapatite" in Andersson $\mathrm{O} \mathrm{H}$ and Yli-Urpo A (Eds.) Bioceramics (London: Butterworth-Heinemann) 1994, pp. 17-22

4. Von Recum AF. Handbook of Biomaterials Evaluation (London: Taylor \& Francis) 1998.

5. Lawrence J, Li L. J. Phys. D 1999; 32: 1075-82

6. Lawrence J, Li L. J. Phys. D 1999; 32: 2311-18

7. Lawrence J, Li L. Appl. Surf. Sci. 2000; 154/155: 664-9.

8. Lawrence J, Li L. Mater. Sci. Eng. A 2001; 303: 142-9.

9. Dausinger F. Proceedings of ECLAT'90: Laser Treatment of Materials, September 1990, Erlangen, Germany, 1991. vol 1, pp. 1-14.

10. Shuttleworth S. App. Surf. Sci. 1996; 96-98: 513-7.

11. Dyer PE, Gonzalo J, Key PE Sands D, Schmidt MJJ. App. Surf. Sci. 1997; 110: 345-9.

12. Chen X, Lotshaw WT, Ortiz AL, Staver PR, Erikson CE. J. of Laser Apps. 1996; 8: 233-9.

13. Bradley L, Li L, Stott FH. App. Surf. Sci. 1998; 138-139: 522-8.

14. Lawrence J, Li L. Mater. Sci. Eng. A 2000; 284: 93-102.

15. Lawrence J, Li L. Mater. Sci. Eng. A 2000; 287: 25-9.

16. Bäuerle D. Laser Processing and Chemistry (Berlin: Springer) 1996. 
17. Andrew JE, Dyer PE, Forster D, Key PH. Appl. Phys. Lett. 1983; 43: 717-22.

18. Schmidt MJJ, Li L, Spencer JT. App. Surf. Sci. 1998; 138-139: 378-84.

19. Lawrence J, Li L. Optics Laser Tech. 2000: 32: 11-4.

20. Lawrence J, Johnston EP, Li L. J. Phys. D 2000; 33: 745-7.

21. Lawrence J, Li L. Appl. Surf. Sci. 2000; 168: 71-4.

22. Lawrence J. PhD Thesis, UMIST, 1999.

23. Dyer PE, Sidhu J. J. Appl. Phys. 1985; 57: 1420-2.

24. Dyer PE, Jenkins SD, Sidhu J. Appl. Phys. Lett. 1986; 49: 453-4. 


\section{List of Figs.}

Fig. 1. Removal rate per shot, $h$, as a function of $\mathrm{CO}_{2}$ laser fluence, $E$.

Fig. 2. Removal rate per shot, $h$, as a function of Nd:YAG laser fluence, $E$.

Fig. 3. Removal rate per shot, $h$, as a function of HPDL fluence, $E$.

Fig. 4. Development of the meltpool in relation to laser beam spot size with time for irradiances of 2 and $3 \mathrm{~kW} / \mathrm{cm}^{2}$ using the $\mathrm{CO}_{2}$ laser.

Fig. 5. Development of the meltpool in relation to laser beam spot size with time for irradiances of 2 and $3 \mathrm{~kW} / \mathrm{cm}^{2}$ using the $\mathrm{Nd}: \mathrm{YAG}$ laser.

Fig. 6. Development of the meltpool in relation to laser beam spot size with time for irradiances of 2 and $3 \mathrm{~kW} / \mathrm{cm}^{2}$ using the HPDL. 
Fig. 1

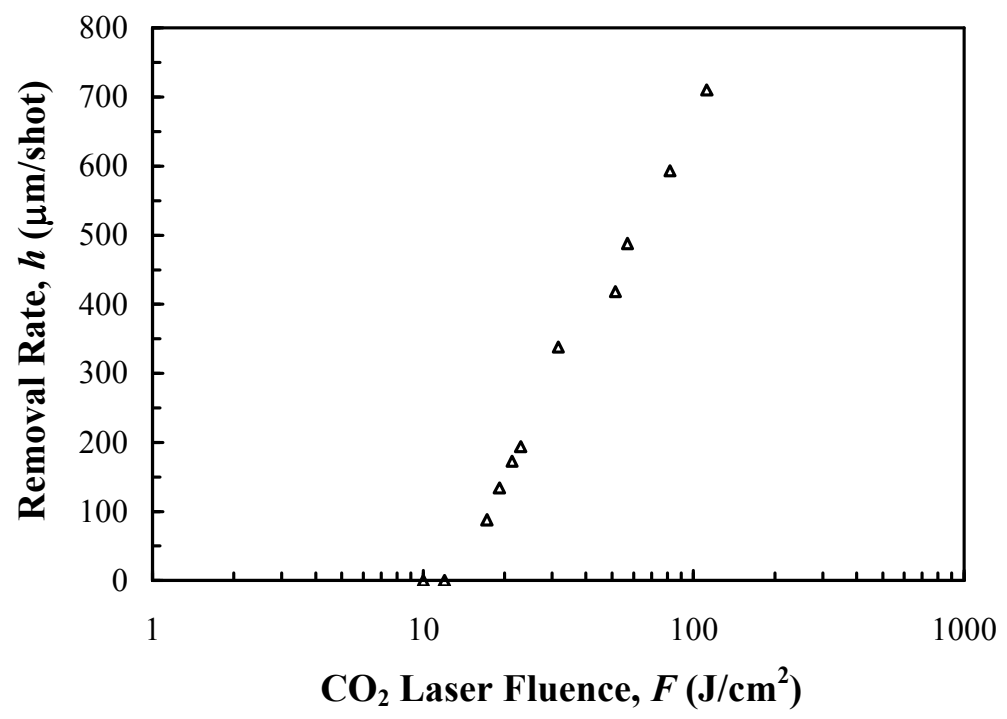


Fig. 2

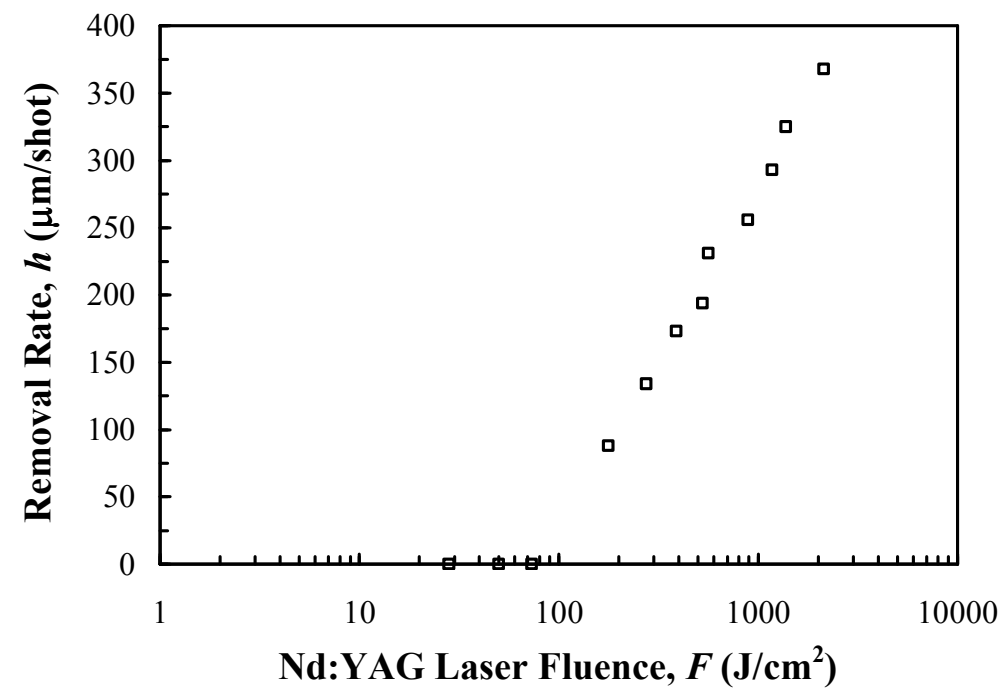


Fig. 3

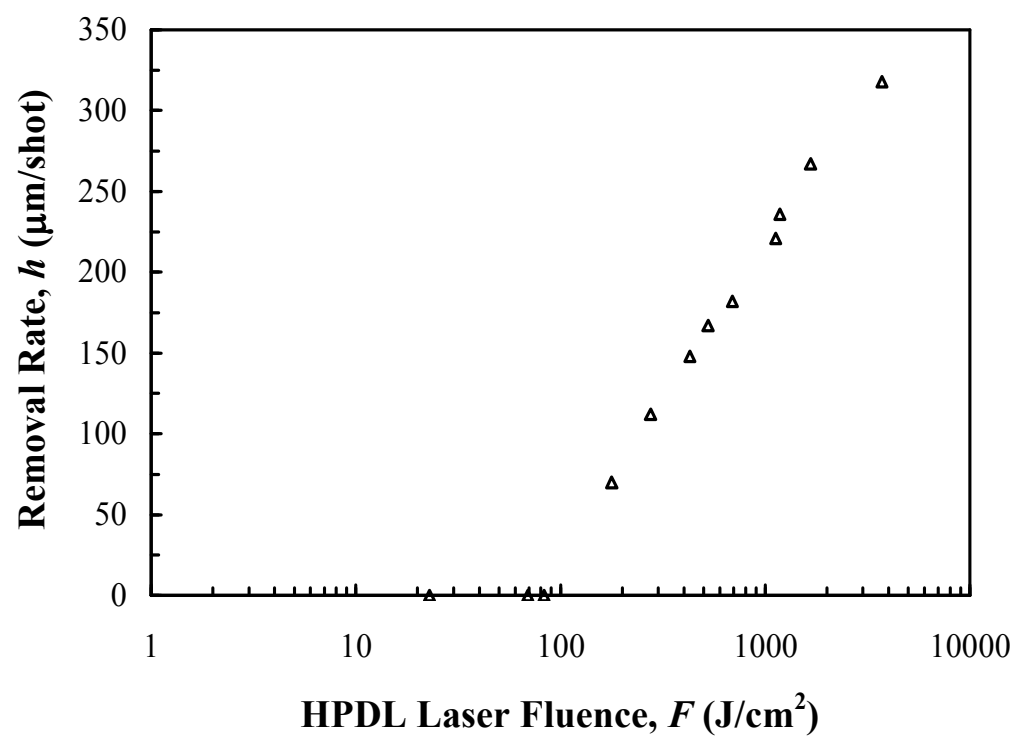


Fig. 4

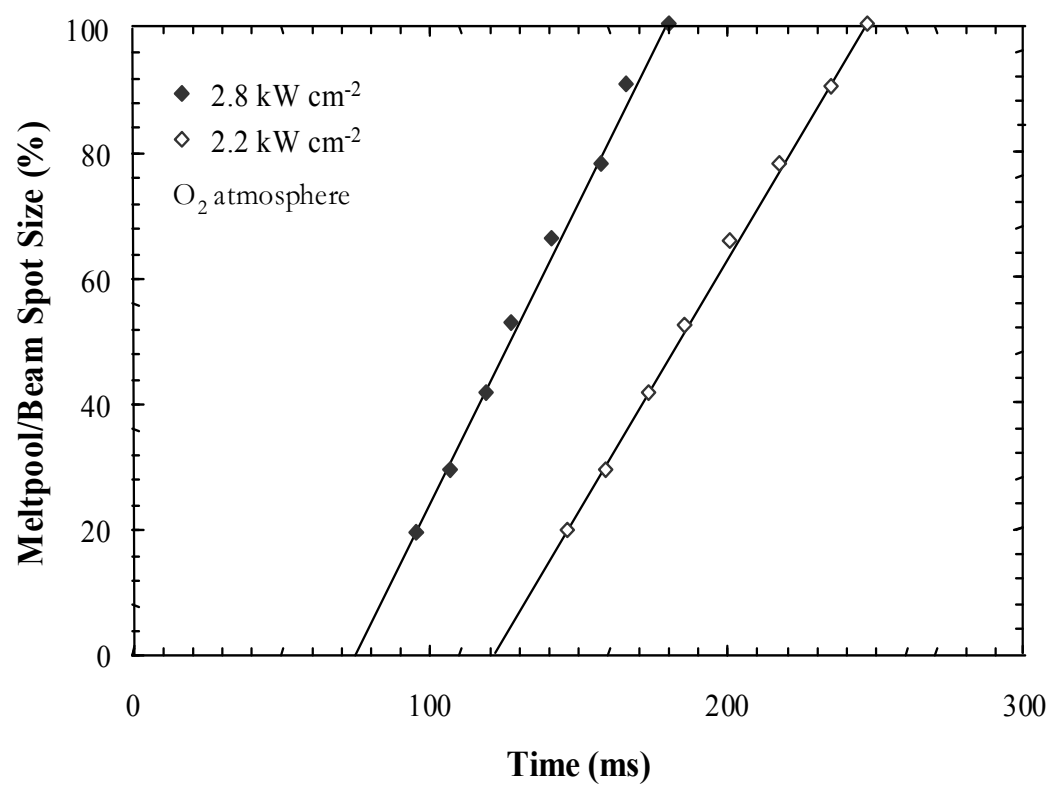


Fig. 5

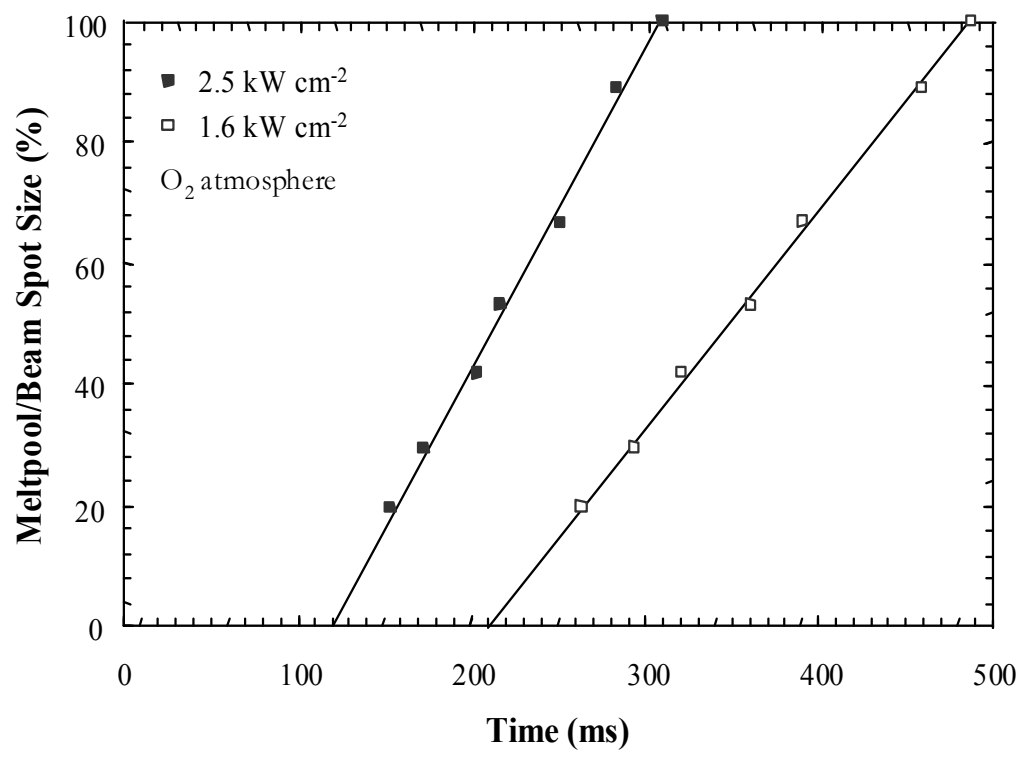


Fig. 6

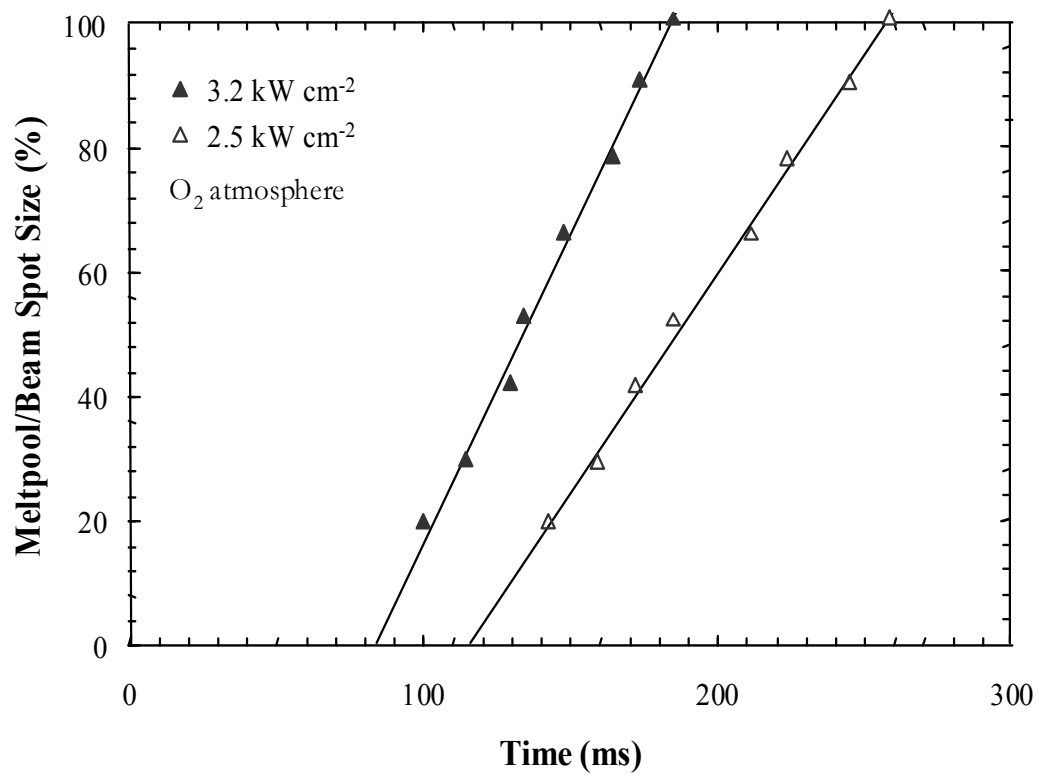




\section{$\underline{\text { List of Tables }}$}

Table 1. Laser interaction properties and effects for the alpha-alumina bioceramic. 


\section{$\underline{\text { Table } 1}$}

\begin{tabular}{lccc}
\hline \multirow{2}{*}{ Property/Effect } & \multicolumn{3}{c}{ Laser Type } \\
& $\begin{array}{c}\text { NO }{ }_{2} \\
(\lambda=10.6 \mu \mathrm{m})\end{array}$ & $\begin{array}{c}\text { Nd:YG } \\
(\lambda=1.06 \mu \mathrm{m})\end{array}$ & $\begin{array}{c}\text { HPDL } \\
(\lambda=940 \mathrm{~nm})\end{array}$ \\
\hline Minimum fluence threshold, $F_{T}$ & $12.36 \mathrm{~J} / \mathrm{cm}^{2}$ & $73.89 \mathrm{~J} / \mathrm{cm}^{2}$ & $83.43 \mathrm{~J} / \mathrm{cm}^{2}$ \\
Absorption length, $1 / \alpha$ & $303 \pm 23 \mu \mathrm{m}$ & $106 \pm 7 \mu \mathrm{m}$ & $88 \pm 4 \mu \mathrm{m}$ \\
Absorptivity & $76 \pm 5 \%$ & $68 \pm 5 \%$ & $63 \pm 5 \%$ \\
Thermal loading, $\gamma$ & $397.92 \mathrm{~J} / \mathrm{cm}^{3}$ & $6.65 \mathrm{~kJ} / \mathrm{cm}^{3}$ & $9.19 \mathrm{~kJ} / \mathrm{cm}^{3}$ \\
Absorption coefficient, $\alpha$ & $33.16 \mathrm{~cm}^{-1}$ & $90.11 \mathrm{~cm}^{-1}$ & $110.28 \mathrm{~cm}^{-1}$ \\
Meltpool propagation speed, $V_{m / s}$ & $0.87 \%(2.8 \mathrm{~kW})$ & $0.72 \%(2.5 \mathrm{~kW})$ & $0.96 \%(3.2 \mathrm{~kW})$ \\
& $0.73 \%(2.2 \mathrm{~kW})$ & $0.47 \%(1.6 \mathrm{~kW})$ & $0.79 \%(2.5 \mathrm{~kW})$ \\
Melt depth (450 W, $1200 \mathrm{~mm} / \mathrm{min})$ & $0.95 \mathrm{~mm}$ & $0.53 \mathrm{~mm}$ & $0.38 \mathrm{~mm}$ \\
\hline
\end{tabular}

